\title{
Integrative genomic analyses of secreted protein acidic and rich in cysteine and its role in cancer prediction
}

\author{
BO WANG ${ }^{1}$, KAI CHEN $^{1}$, WENMING XU ${ }^{2}$, DI CHEN ${ }^{1}$, WEI TANG $^{1}$ and TIAN-SONG XIA ${ }^{3}$ \\ Departments of ${ }^{1}$ Medical Oncology and ${ }^{2}$ Internal Medicine, Huangpu Division of the First Affiliated Hospital, \\ Sun Yat-sen University, Guangzhou, Guangdong 510700; ${ }^{3}$ Department of Breast Surgery, The First \\ Affiliated Hospital of Nanjing Medical University, Nanjing, Jiangsu 210029, P.R. China
}

Received May 10, 2013; Accepted January 22, 2014

DOI: $10.3892 / \mathrm{mmr} .2014 .2339$

\begin{abstract}
Secreted protein acidic and rich in cysteine (SPARC), also termed osteonectin or basement-membrane-40 (BM-40), is a matrix-associated protein that elicits changes in cell shape, inhibits cell-cycle progression and affects the synthesis of extracellular matrix (ECM). The final mature SPARC protein has 286 amino acids with three distinct domains, including an NH2-terminal acidic domain (NT), follistatin-like domain (FS) and C terminus domain (EC). The present study identified SPARC genes from 14 vertebrate genomes and revealed that SPARC existed in all types of vertebrates, including fish, amphibians, birds and mammals. In total, 21 single nucleotide polymorphisms (SNPs) causing missense mutations were identified, which may affect the formation of the truncated form of the SPARC protein. The human SPARC gene was found to be expressed in numerous tissues or organs, including in the bone marrow, whole blood, lymph node, thymus, brain, cerebellum, retina, heart, smooth muscle, skeletal muscle, spinal cord, intestine, colon, adipocyte, kidney, liver, pancreas, thyroid, salivary gland, skin, ovary, uterus, placenta, cervix and prostate. When searched in the PrognoScan database, the human SPARC gene was also found to be expressed in bladder, blood, breast, glioma, esophagus, colorectal, head and neck, ovarian, lung and skin cancer tissues. It was revealed that the association between the expression of SPARC and prognosis varied in different types of cancer, and even in the same cancer from different
\end{abstract}

Correspondence to: $\mathrm{Dr}$ Bo Wang, Department of Medical Oncology, Huangpu Division of the First Affiliated Hospital, Sun Yat-sen University, 107 Yanjiangxi Road, Guangzhou, Guangdong 510700, P.R. China

E-mail: wangbo68377@sina.com

Dr Tian-Song Xia, Department of Breast Surgery, The First Affiliated Hospital of Nanjing Medical University, 300 Guangzhou Road, Nanjing, Jiangsu 210029, P.R. China

E-mail: xiatsswms@163.com

Key words: SPARC, comparative genomics, cancer, prognosis, meta-analysis databases. It implied that the function of SPARC in these tumors may be multidimensional, functioning not just as a tumor suppressor or oncogene.

\section{Introduction}

Secreted protein acidic and rich in cysteine (SPARC), also termed as osteonectin or basement-membrane-40, is a matrix-associated protein that elicits changes in cell shape, inhibits cell-cycle progression and affects the synthesis of extracellular matrix (ECM) (1). The human SPARC gene was initially cloned from a human placenta cDNA library (2). The final mature SPARC protein has 286 amino acids with three distinct domains, including an $\mathrm{NH} 2$-terminal acidic domain (NT), follistatin-like domain (FS) and C terminus domain (EC). The NT domain, spanning the first 52 amino acids (Ala1-Glu52), binds hydroxyapatite and calcium ions. This is followed by FS, which comprises the next 85 amino acids (Asn53-Pro137). This region contains several internal disulfide bonds that stabilize two weakly interacting nodules. The third domain, the EC, is 149 amino acids in length (Cys138-Ile286). It contains two EF-hand motifs that bind calcium with high affinity and is comprised almost entirely of $\beta$-helices.

SPARC binds fibrillar collagen and basal lamina collagen IV (3) and is associated with ECM assembly and fibrosis (4). SPARC has also been demonstrated to regulate the activity of matrix metalloproteinases (MMPs), a family of enzymes considered to be the primary mediators of ECM proteolysis and turnover. SPARC has also been demonstrated to modulate growth factor signaling mediated by cell surface receptors, including vascular endothelial growth factor receptor, basic fibroblast growth factor and transforming growth factor $\beta 1$ (5). SPARC is also involved in activating odontoblasts during tooth development (6). SPARC upregulation in endothelial cells and fibroblasts may contribute to compensatory signaling for controlling angiogenesis (7).

Numerous clinical studies have revealed a correlation between SPARC expression, malignant progression and patient survival $(8,9)$. However, SPARC has demonstrated seemingly contradictory effects on tumor progression in clinical correlative studies and in animal models (10-13). The capacity of SPARC to dictate the tumorigenic phenotype has 
been attributed to its effects on the bioavailability and signaling of integrins and growth factors/chemokines. These molecular pathways contribute to a number of physiological events affecting malignant progression, including ECM remodeling, angiogenesis, immune modulation and metastasis (14-17). Thus, comprehensive investigation regarding whether SPARC is involved in various types of tumor formations is required.

In the present study, SPARC genes from humans, chimpanzees, macaques, orangutans, dogs, cows, horses, mice, rats, opossums, chickens, western clawed frog, zebrafish and fugu were identified by comparative genomic analyses. Conserved transcription factor-binding sites within promoter regions of human SPARC genes were then searched. The expression data, functional relevant single nucleotide polymorphisms (SNPs) and comparative proteomic analyses were conducted. Furthermore, meta-analysis of the prognostic value of SPARC genes in various types of cancer was also performed.

\section{Materials and methods}

Identification of novel SPARC genes in vertebrate genomes and integrative genomic analyses. SPARC genes were searched in the genome sequences of humans (Homo sapiens), chimpanzees (Pan troglodytes), macaques (Macaca mulatta), orangutans (Pongo pygmaeus), dogs (Canis familiaris), cows (Bos taurus), horses (Equus caballus), mice (Mus musculus), rats (Rattus norvegicus), opossums (Monodelphis domestica), chickens (Gallus gallus), frog (Xenopus tropicalis), zebrafish (Danio rerio) and fugu (Takifugu rubripes) by the method described prior to using the human SPARC (NM_003118.3) as queries. The assemblies used were human NCBI 36, chimpanzee CHIMP2.1, macaque MMUL 1.0, orangutan PPYG2, dog Canfam 2.0, cow Btau_4.0, horse Equ Cab 2, mouse NCBI m37, rat RGSC 3.4, opossum monDom5, chicken WASHUC2, frog JGI 4.1, zebrafish Zv8 and fugu FUGU 4.0. The identified putative SPARC genes were BLASTed against the nr database of GenBank to confirm that the best hits were SPARC genes (18-21). Conserved transcription factor-binding sites within promoter regions of the human SPARC gene was obtained from SABiosciences' proprietary database which combines Text Mining Application and data from the UCSC Genome Browser.

Comparative proteomic analyses of SPARC proteins. The amino acid sequences of SPARC were deduced from the identified SPARC genes and aligned using Clustal X 1.8 software (22). The phylogenetic tree of SPARC was obtained using maximum likelihood (ML; PHYML v2.4.4) (23) and neighbor-joining (NJ; MEGA 3.0) (24) methods, and the reliability of the tree was evaluated by the bootstrap method with 1,000 replications. The program Codeml implemented in the PAML 3.14 b software package was used to investigate whether Ikaros proteins are under positive selection (25). Six models of codon substitution, one-ratio (M0), NearlyNeutral (M1a), PositiveSelection (M2a), discrete (M3), $\beta$ (M7) and $\beta$ and $\omega$ (M8) were used in the analysis (26).

Functional relevant SNP evaluation of the humanSPARCgene. Functional relevant SNPs of the human SPARC gene were identified as previously described (18-21). The SNPs were extracted from Ensembl (http://www.ensembl.org) and NCBI's SNPdb (http://www.ncbi.nlm.nih.gov). The SNPs that were able to disrupt exonic splicing enhancer/exonic splicing silencer (ESE/ESS) motifs and cause missense mutations were also identified.

In silico expression analyses of the human SPARC gene. Expressed sequence tags (ESTs) derived from the human SPARC gene were searched for using the BLAST programs as previously described (27-30). The human SPARC gene (NM_003118) was used as a query sequence for the BLAST programs. The expression profiles for normal human tissues were obtained from GeneAnnot (31) and ArrayExpress (32). Northern analysis of NCBI's uniGene dataset was also extracted (18-21). Furthermore, the protein expression of SPARC was obtained from the Systematic Protein Investigative Research Environment (SPIRE) (33) and the Model Organism Protein Expression Database (MOPED) (34).

Meta-analysis of the prognostic value of the SPARC gene in cancer. A database named 'PrognoScan' has been developed (35). This database is a large collection of publicly available cancer microarray datasets with clinical annotation, as well as a tool for assessing the biological association between gene expression and prognosis. PrognoScan employs the minimum P-value approach for grouping patients for survival analysis. PrognoScan provides a powerful platform for evaluating potential tumor markers and therapeutic targets and is publicly accessible at http://gibk21.bio.kyutech.ac.jp/PrognoScan/index.html. The human SPARC gene was inputted as queries and the data were collected for analysis.

\section{Results}

Comparative proteomics of SPARC proteins identified in vertebrate genomes. The SPARC genes were identified in the genome sequences of humans, chimpanzees, macaques, orangutans, dogs, cows, horses, mice, rats, opossums, chickens, western clawed frog, zebrafish and fugu. The refined phylogenetic trees using the identified SPARC proteins amino acid sequences by ML and NJ methods were almost identical (Fig. 1). The present study was unable to identify any site under positive selection, with any of the six models, in the SPARC proteins. Instead, the SPARC proteins were observed to be under purifying selection (data not shown).

Expression profile of the human SPARC gene. By searching for EST sequences, the human SPARC gene was found to be expressed in the eye, placenta, fetal brain, neuroblastoma, fetal liver and Lupski dorsal root ganglion. The investigation of available microarray experiments and 'virtual Northern blot' demonstrated a predominant expression of SPARC in the bone marrow, whole blood, lymph node, thymus, brain, cerebellum, retina, heart, smooth muscle, skeletal muscle, spinal cord, intestine, colon, adipocyte, kidney, liver, pancreas, thyroid, salivary gland, skin, ovary, uterus, placenta, cervix and prostate. When searched in the PrognoScan database, the human SPARC gene was also revealed to be expressed in 


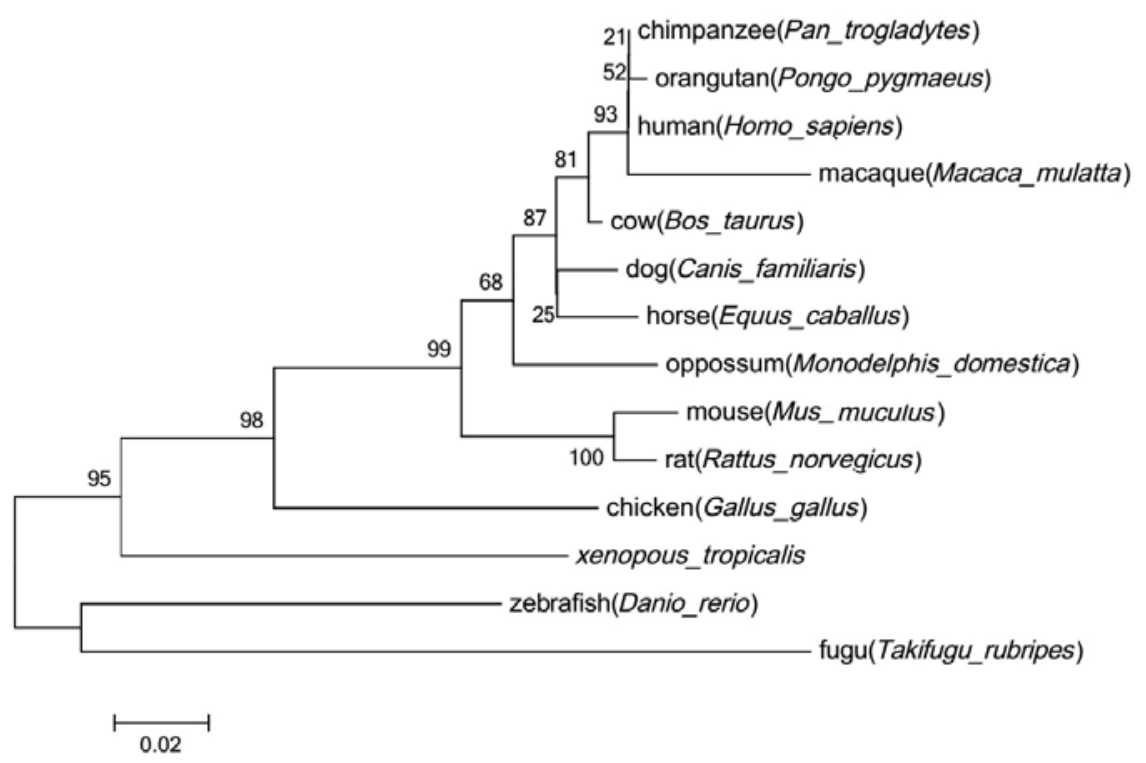

Figure 1. Phylogentic analysis of SPARC. SPARC genes were identified in the genome sequences of humans, chimpanzees, macaques, orangutans, dogs, cows, horses, mouses, rats, opossums, chickens, western clawed frog, zebrafish and fugu. The phylogenetic tree of the SPARC gene was obtained using maximum likelihood and neighbor-joining methods. It appeared that the primate SPARC gene clustered into one group, different from other SPARC genes. SPARC, secreted protein acidic and rich in cysteine.

bladder, blood, brain, breast, colorectal, eye, lung, ovarian, prostate, renal, skin and soft tissue cancer tissues. Among the protein expression databases SPIRE and MOPED, SPARC protein was highly expressed in blood plasma, blood monocyte, kidney HEK-293, liver and liver HuH-7 cancer cells; however, low levels of SPARC protein expression were observed in blood erythroleukemia, blood neutrophil, blood B-lymphocyte, blood T-lymph Jurkat, kidney urine, lung alveolar lavage, pancreatic cancer and prostate cancer tissues.

Comparative genomics of the human SPARC gene. Nkx2-5, Brachyury PPAR- $\alpha$, AML1a and p53 regulatory transcription factor binding sites were identified in the SPARC gene upstream (promoter) region.

Functional relevant SNP evaluation of the human SPARC gene. In total, 471 available SNPs were identified in the human SPARC gene. Among these, 23 SNPs were functionally relevant, including two available alleles, which disrupted an existing ESE and 21 SNPs causing missense mutations (Table I).

Meta-analysis of the prognostic value of the human SPARC gene in cancer. When provided with the gene, PrognoScan exhibits a summary in table format of tests for the gene with columns for dataset, cancer type, subtype, end point, cohort, contributor, array type, probe ID, number of patient, optimal cut point, Pmin and Pcor. Among the databases that detected the expression of the SPARC gene, 27 out of 136 tests demonstrated an association between microarray expression in the SPARC gene and cancer prognosis (bladder cancer $0 / 4$, blood cancer $5 / 18$, brain cancer $0 / 8$, breast cancer $4 / 38$, colorectal cancer $8 / 18$, eye cancer $2 / 2$, lung cancer
$3 / 31$, ovarian cancer $4 / 13$, prostate cancer $1 / 1$, renal cancer $0 / 1$, skin cancer $0 / 2$ and soft tissue cancer $0 / 2$ ) with a $5 \%$ significance level (Table II, 36-50). Among the five types of blood cancer, a higher expression of the SPARC gene was associated with a poor survival rate and was found in three acute myeloid leukemia (AML) cases (GSE12417-GPL96, GSE12417-GPL96 and GSE12417-GPL570). However, in B-cell lymphoma (GSE4475) and diffuse large B-cell lymphoma (DLBCL; TABM-346) cases, a lower expression of the SPARC gene was associated with a poor survival rate. Among the four types of breast cancer, a higher expression of the SPARC gene was associated with a poor survival rate and was identified in two cases (GSE11121 and E-TABM-158). However, a lower expression of the SPARC gene was associated with a poor survival rate in another two cases of breast cancer (GSE3494-GPL96). The present study revealed that a higher expression of the SPARC gene was associated with a poor survival rate in all eight colorectal cancer cases. In lung cancer cases, a higher expression of the SPARC gene was associated with a poor survival rate in all three types of lung cancer, including adenocarcinoma and non-small cell lung cancer (NSCLC). In addition, a lower expression of the SPARC gene was associated with a poor survival rate in four cases of ovarian cancer, two cases of eye cancer and one case of prostate cancer.

\section{Discussion}

SPARC, also known as osteonectin or BM-40, is a matrix-associated protein that elicits changes in cell shape, inhibits cell-cycle progression and affects the synthesis of the extracellular matrix (ECM) (1). In the present study, additional SPARC genes from 13 other vertebrate genomes 
Table I. Functional relevant SNP evaluation of the human SPARC gene.

\begin{tabular}{|c|c|c|c|c|}
\hline SNP ID & Chr 5 position & Sequence & Type & $\begin{array}{c}\text { Amino acid } \\
\text { change }\end{array}$ \\
\hline rs707157 & $151047108(-)$ & TGCGGA/G/TACTGG & Missense & $\mathrm{ND} / \mathrm{Y}$ \\
\hline rs 1053296 & $151047111(-)$ & GCATGC/GGGGAC & Missense & $\mathrm{R} / \mathrm{D}$ \\
\hline rs11542492 & $151049293(-)$ & TGCCAC/TAAAGT & Missense & $\mathrm{T} / \mathrm{I}$ \\
\hline rs11542497 & $151054219(-)$ & CCTGCA/CTGATG & Missense & $\mathrm{H} / \mathrm{P}$ \\
\hline rs11542498 & $151054198(-)$ & GGTGGA/GAGAAA & Missense & $\mathrm{E} / \mathrm{G}$ \\
\hline rs41290587 & $151051255(+)$ & AGGGAT/CCTGTA & Missense & $/ \mathrm{N} / \mathrm{S}$ \\
\hline rs7433231 & $151045923(+)$ & TTACCC/TGTCAA & Missense & $\mathrm{R} / \mathrm{G}$ \\
\hline rs113617771 & $151052711(+)$ & СTCTTC/TGGTTT & Missense & $\mathrm{K} / \mathrm{E}$ \\
\hline rs141567625 & $151045999(+)$ & TCGAAG/TTCCCG & Missense & $\mathrm{E} / \mathrm{D}$ \\
\hline rs142207246 & $151051184(+)$ & GCACAC/TGCACA & Missense & $\mathrm{M} / \mathrm{V}$ \\
\hline rs 142378176 & $151051171(+)$ & TGGTGA/GGGTCC & Missense & $\mathrm{P} / \mathrm{L}$ \\
\hline rs142717464 & $151043728(+)$ & AAAAGC/TGGGTG & Missense & $\mathrm{H} / \mathrm{R}$ \\
\hline rs 146500464 & $151052741(+)$ & TТСТСС/ТTAСТT & Missense & $\mathrm{R} / \mathrm{G}$ \\
\hline rs147557671 & $151051145(+)$ & AAACTC/TGCCAA & Missense & $\mathrm{K} / \mathrm{E}$ \\
\hline rs185684862 & $151047110(+)$ & AGTCCC/TGCATG & Missense & $\mathrm{Q} / \mathrm{R}$ \\
\hline rs188911380 & $151043660(+)$ & GATGCC/TGAAGC & Missense & $\mathrm{S} / \mathrm{G}$ \\
\hline rs199591638 & $151051252(+)$ & GGCAGG/TGATCT & Missense & $\mathrm{H} / \mathrm{P}$ \\
\hline rs199655940 & $151043747(+)$ & CTCCAC/TGGGGA & Missense & $\mathrm{M} / \mathrm{V}$ \\
\hline rs200777949 & $151047030(+)$ & TACCCA/GCAGCT & Missense & $\mathrm{R} / \mathrm{W}$ \\
\hline rs201797309 & $151049318(+)$ & AGAGTC/TGAAGG & Missense & N/D \\
\hline rs201856432 & $151045950(+)$ & CTGGCC/TGAACT & Missense & $\mathrm{S} / \mathrm{G}$ \\
\hline rs2304049 & $151047100(+)$ & TTCTTG/CAGCCA & ESE & \\
\hline rs11542495 & $151049274(-)$ & GAGGGC/TACCAA & ESE & \\
\hline
\end{tabular}

In total, 471 available SNPs were identified in the human SPARC gene. Among these, 23 SNPs were functionally relevant, including two available alleles, which disrupted an existing ESE and 21 SNPs causing missense mutations. SNP, single nucleotide polymorphism; SPARC, secreted protein acidic and rich in cysteine; ESE, exonic splicing enhancer.

were identified and SPARC was found to exist in all types of vertebrates, including fish, amphibians, birds and mammals. Furthermore, all identified RON proteins contained NT, FS and EC domains. The phylogenetic tree demonstrated that SPARC is separated in the order fish, amphibians, birds and mammals. Primate SPARCs are almost the identical and clustered together. From the alignment and phylogenetic tree, mammalian SPARCs were observed to be conserved among vertebrate genomes, suggesting that the function of SPARC may be important physiologically for all the vertebrates in the long evolutionary process. Furthermore, this process was under purifying selection. It is in accordance with multiple biological functions that have been ascribed to this protein, including its involvement in tissue remodeling (51), morphogenesis $(52,53)$ and bone mineralization (54).

Matrix metalloproteinases (MMP-2, -3, -7 and -13), plasmin and trypsin, have been demonstrated to cleave SPARC in vitro, producing a KGHK-containing fragment $(15,55,56-58)$. The presence of the truncated form of the SPARC protein has been reported in hepatocellular carcinoma samples $(59,60)$ and esophageal carcinoma (61). It appeared that truncated SPARC may have an important pro-angiogenic function in cancer. The present study identified 21 SNPs causing missense mutations, which may affect the formation of the truncated form of the SPARC protein. The effects of these SNPs on the physiological and pathological functions of SPARC requires further investigation.

SPARC was initially identified in bone and endothelial cells $(62,63)$. It is also highly expressed in developing tissues, including the notochord (64), somites (65) and the embryonic skeleton (66), as well as in differentiating chondrocytes (67), megakaryocytes (68) and macrophages (69) at sites of tissue injury. The systematic analysis of SPARC expression in normal tissues and cancer samples has not been well studied. The present study revealed that the human SPARC gene was expressed in numerous tissues and organs, including the bone marrow, whole blood, lymph node, thymus, brain, cerebellum, retina, heart, smooth muscle, skeletal muscle, spinal cord, intestine, colon, adipocyte, kidney, liver, pancreas, thyroid, salivary gland, skin, ovary, uterus, placenta, cervix and prostate.

When searched in the PrognoScan database, the human SPARC gene was also revealed to be expressed in bladder, blood, breast, giloma, esophagus, colorectal, head and neck, ovarian, lung and skin cancer tissues. SPARC is differentially expressed in tumors and its surrounding stroma in various types of cancer in comparison with the normal tissue, yet, its pattern of expression is variable depending on the type of 


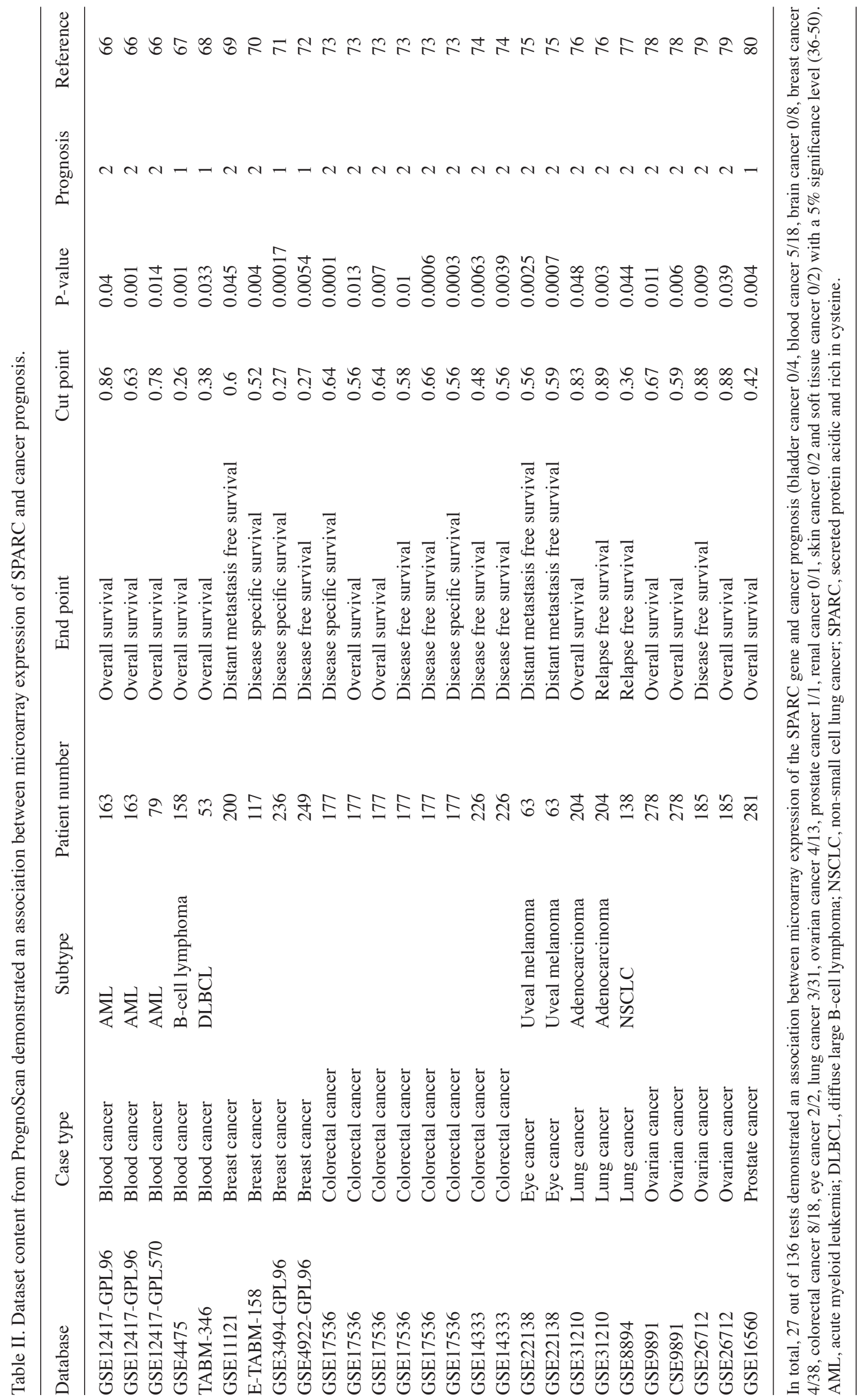


cancer. In total, 27 out of 136 tests demonstrated an association between microarray expression of the SPARC gene and cancer prognosis (bladder cancer $0 / 4$, blood cancer $5 / 18$, brain cancer $0 / 8$, breast cancer $4 / 38$, colorectal cancer $8 / 18$, eye cancer $2 / 2$, lung cancer $3 / 31$, ovarian cancer $4 / 13$, prostate cancer $1 / 1$, renal cancer $0 / 1$, skin cancer $0 / 2$ and soft tissue cancer $0 / 2$ ) with a 5\% significance level.

SPARC mRNA was significantly overexpressed in pancreatic cancer; however, not in cancer of the papilla of Vater (8). SPARC was demonstrated to be associated with drug resistance in ovarian cancer (70). In addition, SPARC induced the migration of glioblastoma cell lines (10) and the downregulation of SPARC expression inhibited cell migration and invasion in malignant gliomas (11). However, other studies suggested that SPARC induced endoplasmic reticulum stress leading to autophagy-mediated apoptosis in neuroblastoma (12), and RNA interference against SPARC promoted the growth of malignant glioma cells (13). The aberrant methylation of SPARC was identified in human laryngeal and hypopharyngeal carcinomas (71). In addition, SPARC was observed to be involved in the transformation of hamster oral mucosa from precancerous lesions to squamous cell carcinoma $(72,73)$. SPARC protein expression was also observed to be markedly induced in the supernatants of co-cultured astrocytes (74). Despite transcriptional silencing by aberrant hypermethylation of the CpG-rich region in endometrial carcinoma, the SPARC protein remained overexpressed (9). Furthermore, microRNA-29a was able to suppress cell proliferation by targeting SPARC in hepatocellular carcinoma (75).

This suggested that the expression of SPARC was associated with the prognosis of numerous types of cancer, including hematological and solid cancers. The underlying mechanisms of SPARC involved in the process of these tumors requires further investigation. It is important to note that the association between the expression of SPARC and prognosis varied in different types of cancer, even in the same cancer from different databases. It implied that the function of SPARC in these tumors may be multidimensional, functioning not just as a tumor suppressor or oncogene.

Nkx2-5, Brachyury PPAR- $\alpha$, AML1a and p53 regulatory transcription factor binding sites were identified in the SPARC gene upstream (promoter) region. Nkx2-5 encodes a homeobox-containing transcription factor. This transcription factor functions during heart formation and development. It was also revealed that Nkx2-5 is the key transcription factor regulating its genomic neighborhoods differently between the tumor types (76). The p53 gene is mutated in approximately half of all types of human tumor. p53 is a transcription factor and its activity gives rise to a variety of cellular outcomes, most notably cell cycle arrest and apoptosis, eliminating cancer-prone cells from the replicative pool (77-79). These two tumor-related transcriptional factors may be involved in the effect of SPARC on various types of tumor.

The present study demonstrated that the association between the expression of SPARC and prognosis varied in different types of cancer. However, the specific functions of SPARC in the majority of tumors remain to be elucidated. Further studies are required to focus on its different behaviors in different types of cancer and its potential relative pathways, including MMPs.

\section{Acknowledgements}

This study was supported in part by the National Natural Science Foundation of China (grant no. 81202077), the Program for Development of Innovative Research Team in the First Affiliated Hospital of NJMU (grant no. IRT-008) and a project Funded by the Priority Academic Program Development of Jiangsu Higher Education Institutions.

\section{References}

1. Bradshaw AD, Graves DC, Motamed $\mathrm{K}$ and Sage EH: SPARC-null mice exhibit increased adiposity without significant differences in overall body weight. Proc Natl Acad Sci USA 100: 6045-6050, 2003.

2. Schwartz RC, Young MF and Tsipouras P: Two RFLPs in the 5 ' end of the human osteonectin (ON) gene. Nucleic Acids Res 16: 9076, 1988.

3. Mayer U, Aumailley M, Mann K, Timpl R and Engel J: Calcium-dependent binding of basement membrane protein BM-40 (osteonectin, SPARC) to basement membrane collagen type IV. Eur J Biochem 198: 141-150, 1991

4. Bradshaw AD: The role of SPARC in extracellular matrix assembly. J Cell Commun Signal 3: 239-246, 2009.

5. Rivera LB and Brekken RA: SPARC promotes pericyte recruitment via inhibition of endoglin-dependent TGF-beta1 activity. J Cell Biol 193: 1305-1319, 2011.

6. Choi BD, Yun SH, Jeong SJ, et al: Expression of thymosin beta4 in odontoblasts during mouse tooth development. Int J Mol Med 29: 841-847, 2012.

7. Nonogaki S, Campos HG, Butugan O, et al: Markers of vascular differentiation, proliferation and tissue remodeling in juvenile nasopharyngeal angiofibromas. Exp Ther Med 1: 921-926, 2010.

8. Prenzel KL, Warnecke-Eberz U, Xi H, et al: Significant overexpression of SPARC/osteonectin mRNA in pancreatic cancer compared to cancer of the papilla of Vater. Oncol Rep 15: 1397-1401, 2006.

9. Rodriguez-Jiménez FJ, Caldés T, Iniesta P, Vidart JA, Garcia-Asenjo JL and Benito M: Overexpression of SPARC protein contrasts with its transcriptional silencing by aberrant hypermethylation of SPARC CpG-rich region in endometrial carcinoma. Oncol Rep 17: 1301-1307, 2007.

10. Kunigal S, Gondi CS, Gujrati M, et al: SPARC-induced migration of glioblastoma cell lines via uPA-uPAR signaling and activation of small GTPase RhoA. Int J Oncol 29: 1349-1357, 2006.

11. Seno T, Harada H, Kohno S, Teraoka M, Inoue A and Ohnishi T: Downregulation of SPARC expression inhibits cell migration and invasion in malignant gliomas. Int J Oncol 34: 707-715, 2009.

12. Sailaja GS, Bhoopathi P, Gorantla B, et al: The secreted protein acidic and rich in cysteine (SPARC) induces endoplasmic reticulum stress leading to autophagy-mediated apoptosis in neuroblastoma. Int J Oncol 42: 188-196, 2013.

13. Liu H, Xu Y, Chen Y, et al: RNA interference against SPARC promotes the growth of U-87MG human malignant glioma cells. Oncol Lett 2: 985-990, 2011.

14. Al Saleh S, Sharaf LH and Luqmani YA: Signalling pathways involved in endocrine resistance in breast cancer and associations with epithelial to mesenchymal transition (Review). Int J Oncol 38: 1197-1217, 2011.

15. Zhang JL, Chen GW, Liu YC, et al: Secreted protein acidic and rich in cysteine (SPARC) suppresses angiogenesis by down-regulating the expression of VEGF and MMP-7 in gastric cancer. PLoS One 7: e44618, 2012.

16. Arnold SA, Rivera LB, Carbon JG, et al: Losartan slows pancreatic tumor progression and extends survival of SPARC-null mice by abrogating aberrant TGFbeta activation. PLoS One 7: e31384, 2012.

17. Said N, Frierson HF, Sanchez-Carbayo M, Brekken RA and Theodorescu D: Loss of SPARC in bladder cancer enhances carcinogenesis and progression. J Clin Invest 123: 751-766, 2013.

18. Yang L, Luo Y and Wei J: Integrative genomic analyses on Ikaros and its expression related to solid cancer prognosis. Oncol Rep 24: 571-577, 2010

19. Yang L, Luo Y, Wei J and He S: Integrative genomic analyses on IL28RA, the common receptor of interferon-lambda1, -lambda2 and -lambda3. Int J Mol Med 25: 807-812, 2010. 
20. Yang L, Wei J and He S: Integrative genomic analyses on interferon-lambdas and their roles in cancer prediction. Int $\mathrm{J}$ Mol Med 25: 299-304, 2010.

21. Yu H, Yuan J, Xiao C and Qin Y: Integrative genomic analyses of recepteur d'origine nantais and its prognostic value in cancer. Int $\mathrm{J}$ Mol Med 31: 1248-1254, 2013.

22. Thompson JD, Gibson TJ, Plewniak F, Jeanmougin F and Higgins DG: The CLUSTAL X windows interface: flexible strategies for multiple sequence alignment aided by quality analysis tools. Nucleic Acids Res 25: 4876-4882, 1997.

23. Guindon S, Lethiec F, Duroux P and Gascuel O: PHYML Online - a web server for fast maximum likelihood-based phylogenetic inference. Nucleic Acids Res 33: W557-W559, 2005.

24. Kumar S, Tamura K and Nei M: MEGA3: Integrated software for Molecular Evolutionary Genetics Analysis and sequence alignment. Brief Bioinform 5: 150-163, 2004.

25. Yang Z: PAML: a program package for phylogenetic analysis by maximum likelihood. Comput Appl Biosci 13: 555-556, 1997.

26. Yang Z, Nielsen R, Goldman $N$ and Pedersen AM: Codon-substitution models for heterogeneous selection pressure at amino acid sites. Genetics 155: 431-449, 2000.

27. Katoh Y and Katoh M: Integrative genomic analyses on GLI1: positive regulation of GLI1 by Hedgehog-GLI, TGFbeta-Smads, and RTK-PI3K-AKT signals, and negative regulation of GLI1 by Notch-CSL-HES/HEY, and GPCR-Gs-PKA signals. Int J Oncol 35: 187-192, 2009.

28. Katoh $\mathrm{M}$ and Katoh M: Integrative genomic analyses of WNT11: transcriptional mechanisms based on canonical WNT signals and GATA transcription factors signaling. Int J Mol Med 24: 247-251, 2009.

29. Katoh M and Katoh M: Transcriptional mechanisms of WNT5A based on NF-kappaB, Hedgehog, TGFbeta, and Notch signaling cascades. Int J Mol Med 23: 763-769, 2009.

30. Katoh $\mathrm{M}$ and Katoh M: Transcriptional regulation of WNT2B based on the balance of Hedgehog, Notch, BMP and WNT signals. Int J Oncol 34: 1411-1415, 2009.

31. Chalifa-Caspi V, Yanai I, Ophir R, et al: GeneAnnot: comprehensive two-way linking between oligonucleotide array probesets and GeneCards genes. Bioinformatics 20: 1457-1458, 2004.

32. Parkinson H, Sarkans U, Shojatalab M, et al: ArrayExpress - a public repository for microarray gene expression data at the EBI. Nucleic Acids Res 33: D553-D555, 2005.

33. Kolker E, Higdon R, Morgan P, et al: SPIRE: Systematic protein investigative research environment. J Proteomics 75: 122-126, 2011.

34. Kolker E, Higdon R, Haynes W, et al: MOPED: Model Organism Protein Expression Database. Nucleic Acids Res 40: D1093-D1099, 2012.

35. Mizuno H, Kitada K, Nakai K and Sarai A: PrognoScan: a new database for meta-analysis of the prognostic value of genes. BMC Med Genomics 2: 18, 2009.

36. Metzeler KH, Hummel M, Bloomfield CD, et al: An 86-probe-set gene-expression signature predicts survival in cytogenetically normal acute myeloid leukemia. Blood 112: 4193-4201, 2008

37. Hummel M, Bentink S, Berger $\mathrm{H}$, et al: A biologic definition of Burkitt's lymphoma from transcriptional and genomic profiling. N Engl J Med 354: 2419-2430, 2006.

38. Jardin F, Jais JP, Molina TJ, et al: Diffuse large B-cell lymphomas with CDKN2A deletion have a distinct gene expression signature and a poor prognosis under R-CHOP treatment: a GELA study. Blood 116: 1092-1104, 2010

39. Schmidt M, Böhm D, von Torne C, et al: The humoral immune system has a key prognostic impact in node-negative breast cancer. Cancer Res 68: 5405-5413, 2008.

40. Chin K, DeVries S, Fridlyand J, et al: Genomic and transcriptional aberrations linked to breast cancer pathophysiologies. Cancer Cell 10: 529-541, 2006.

41. Miller LD, Smeds J, George J, et al: An expression signature for p53 status in human breast cancer predicts mutation status, transcriptional effects, and patient survival. Proc Natl Acad Sci USA 102: $13550-13555,2005$

42. Ivshina AV, George J, Senko O, et al: Genetic reclassification of histologic grade delineates new clinical subtypes of breast cancer. Cancer Res 66: 10292-10301, 2006.

43. Smith JJ, Deane NG, Wu F, et al: Experimentally derived metastasis gene expression profile predicts recurrence and death in patients with colon cancer. Gastroenterology 138: 958-968, 2010.

44. Jorissen RN, Gibbs P, Christie M, et al: Metastasis-associated gene expression changes predict poor outcomes in patients with Dukes stage B and C colorectal cancer. Clin Cancer Res 15: 7642-7651, 2009.
45. Laurent C, Valet F, Planque N, et al: High PTP4A3 phosphatase expression correlates with metastatic risk in uveal melanoma patients. Cancer Res 71: 666-674, 2011.

46. Okayama H, Kohno T, Ishii Y, et al: Identification of genes upregulated in ALK-positive and EGFR/KRAS/ALK-negative lung adenocarcinomas. Cancer Res 72: 100-111, 2012.

47. Lee ES, Son DS, Kim SH, et al: Prediction of recurrence-free survival in postoperative non-small cell lung cancer patients by using an integrated model of clinical information and gene expression. Clin Cancer Res 14: 7397-7404, 2008.

48. Tothill RW, Tinker AV, George J, et al: Novel molecular subtypes of serous and endometrioid ovarian cancer linked to clinical outcome. Clin Cancer Res 14: 5198-5208, 2008.

49. Bonome T, Levine DA, Shih J, et al: A gene signature predicting for survival in suboptimally debulked patients with ovarian cancer Cancer Res 68: 5478-5486, 2008.

50. Sboner A, Demichelis F, Calza S, et al: Molecular sampling of prostate cancer: a dilemma for predicting disease progression. BMC Med Genomics 3: 8, 2010

51. McCurdy SM, Dai Q, Zhang J, et al: SPARC mediates early extracellular matrix remodeling following myocardial infarction. Am J Physiol Heart Circ Physiol 301: H497-H505, 2011.

52. Cheng L, Sage EH and Yan Q: SPARC fusion protein induces cellular adhesive signaling. PLoS One 8: e53202, 2013.

53. Nakamura K, Nakano S, Miyoshi T, Yamanouchi K, Matsuwaki T and Nishihara M: Age-related resistance of skeletal muscle-derived progenitor cells to SPARC may explain a shift from myogenesis to adipogenesis. Aging (Albany NY) 4: 40-48, 2012.

54. Pataquiva-Mateus AY, Wu HC, Lucchesi C, Ferraz MP, Monteiro FJ and Spector M: Supplementation of collagen scaffolds with SPARC to facilitate mineralization. J Biomed Mater Res B Appl Biomater 100: 862-870, 2012.

55. Li B, Li F, Chi L, Zhang L and Zhu S: The expression of SPARC in human intracranial aneurysms and its relationship with MMP-2/-9. PLoS One 8: e58490, 2013.

56. Seet LF, Tong L, Su R and Wong TT: Involvement of SPARC and MMP-3 in the pathogenesis of human pterygium. Invest Ophthalmol Vis Sci 53: 587-595, 2012.

57. Patterson J and Hubbell JA: SPARC-derived protease substrates to enhance the plasmin sensitivity of molecularly engineered PEG hydrogels. Biomaterials 32: 1301-1310, 2011.

58. Weaver MS, Sage EH and Yan Q: Absence of SPARC in lens epithelial cells results in altered adhesion and extracellular matrix production in vitro. J Cell Biochem 97: 423-432, 2006.

59. Lin ZY and Chuang WL: Genes responsible for the characteristics of primary cultured invasive phenotype hepatocellular carcinoma cells. Biomed Pharmacother 66: 454-458, 2012.

60. Zhang Y, Yang B, Du Z, et al: Aberrant methylation of SPARC in human hepatocellular carcinoma and its clinical implication. World J Gastroenterol 18: 2043-2052, 2012.

61. Xue LY, Zou SM, Zheng S, et al: Expressions of the gamma2 chain of laminin-5 and secreted protein acidic and rich in cysteine in esophageal squamous cell carcinoma and their relation to prognosis. Chin J Cancer 30: 69-78, 2011.

62. Termine JD, Kleinman HK, Whitson SW, Conn KM, McGarvey ML and Martin GR: Osteonectin, a bone-specific protein linking mineral to collagen. Cell 26: 99-105, 1981.

63. Mason IJ, Murphy D, Münke M, Francke U, Elliott RW and Hogan BL: Developmental and transformation-sensitive expression of the Sparc gene on mouse chromosome 11. EMBO J 5: 1831-1837, 1986

64. Huynh MH, Sodek K, Lee H and Ringuette M: Interaction between SPARC and tubulin in Xenopus. Cell Tissue Res 317: 313-317, 2004.

65. Sage H, Decker J, Funk S and Chow M: SPARC: a $\mathrm{Ca}^{2+}$-binding extracellular protein associated with endothelial cell injury and proliferation. J Mol Cell Cardiol 21 (Suppl 1): 13-22, 1989.

66. Leboy PS, Shapiro IM, Uschmann BD, Oshima O and Lin D: Gene expression in mineralizing chick epiphyseal cartilage. J Biol Chem 263: 8515-8520, 1988.

67. Aeschlimann D, Kaupp O and Paulsson $\mathrm{M}$ : Transglutaminase-catalyzed matrix cross-linking in differentiating cartilage: identification of osteonectin as a major glutaminyl substrate. J Cell Biol 129: 881-892, 1995.

68. Breton-Gorius J, Clezardin P, Guichard J, et al: Localization of platelet osteonectin at the internal face of the alpha-granule membranes in platelets and megakaryocytes. Blood 79: 936-941, 1992.

69. Komatsubara I, Murakami T, Kusachi S, et al: Spatially and temporally different expression of osteonectin and osteopontin in the infarct zone of experimentally induced myocardial infarction in rats. Cardiovasc Pathol 12: 186-194, 2003. 
70. Yin F, Liu X, Li D, Wang Q, Zhang W and Li L: Bioinformatic analysis of chemokine (C-C motif) ligand 21 and SPARC-like protein 1 revealing their associations with drug resistance in ovarian cancer. Int J Oncol 42: 1305-1316, 2013.

71. He Q, Wei J, Zhang J, et al: Aberrant methylation of secreted protein, acidic and rich in cysteine in human laryngeal and hypopharyngeal carcinoma. Oncol Lett 2: 725-729, 2011.

72. Chen D, Yang K, Mei J, Zhang G, Lv X and Xiang L: Screening the pathogenic genes and pathways related to DMBA (7,12-dimethylbenz[a]anthracene)-induced transformation of hamster oral mucosa from precancerous lesions to squamous cell carcinoma. Oncol Lett 2: 637-642, 2011.

73. Suhr ML, Dysvik B, Bruland O, et al: Gene expression profile of oral squamous cell carcinomas from Sri Lankan betel quid users. Oncol Rep 18: 1061-1075, 2007.

74. Gagliano N, Costa F, Cossetti C, et al: Glioma-astrocyte interaction modifies the astrocyte phenotype in a co-culture experimental model. Oncol Rep 22: 1349-1356, 2009.
75. Zhu XC, Dong QZ, Zhang XF, et al: microRNA-29a suppresses cell proliferation by targeting SPARC in hepatocellular carcinoma. Int J Mol Med 30: 1321-1326, 2012.

76. Ylipää A, Yli-Harja O, Zhang W and Nykter M: A systems biological approach to identify key transcription factors and their genomic neighborhoods in human sarcomas. Chin J Cancer 30: 27-40, 2011.

77. Ren D, Wang M, Guo W, et al: Wild-type p53 suppresses the epithelial-mesenchymal transition and stemness in PC-3 prostate cancer cells by modulating miR-145. Int J Oncol 42: 1473-1481, 2013.

78. Abou-El-Ardat K, Derradji H, de Vos W, et al: Response to low-dose $\mathrm{X}$-irradiation is $\mathrm{p} 53$-dependent in a papillary thyroid carcinoma model system. Int J Oncol 39: 1429-1441, 2011.

79. Stefancikova L, Moulis M, Fabian P, et al: Prognostic impact of p53 aberrations for R-CHOP-treated patients with diffuse large B-cell lymphoma. Int J Oncol 39: 1413-1420, 2011. 